\title{
Existence and Uniqueness of Solutions for a Class of Nonlinear Stochastic Differential Equations
}

\author{
Iryna Volodymyrivna Komashynska \\ Mathematics Department, University of Jordan, Amman 11942, Jordan \\ Correspondence should be addressed to Iryna Volodymyrivna Komashynska; iryna_kom@hotmail.com
}

Received 28 November 2012; Accepted 28 January 2013

Academic Editor: Paul Eloe

Copyright ( 2013 Iryna Volodymyrivna Komashynska. This is an open access article distributed under the Creative Commons Attribution License, which permits unrestricted use, distribution, and reproduction in any medium, provided the original work is properly cited.

\begin{abstract}
By using successive approximation, we prove existence and uniqueness result for a class of nonlinear stochastic differential equations. Moreover, it is shown that the solution of such equations is a diffusion process and its diffusion coefficients are found.
\end{abstract}

\section{Introduction}

Differential equations, which are not solved for the derivative, have found diverse applications in many fields. Examples of equations of this type are Lagrange equations of classical mechanics or Euler equations.

Consideration of real objects under the influence of random factors leads to nonlinear stochastic differential equations, which are not solved for stochastic differential. Such equations were introduced by Kolmanovskii and Nosov in [1] for construction of stochastic analogues of neutral functional differential equations. Works [1-5] were devoted to the problems of existence, uniqueness, and properties of solutions of neutral stochastic differential (delay) equations in finite dimensional spaces. Existence of solutions for such equations in Hilbert spaces was studied in papers [6-9]. In the paper [9] the author considered a stochastic equation without delay. In the monograph of Kolmanovskii and Shaǐkhet [10] conditions were obtained for optimality in control problems for these equations.

In this paper we will study the existence and uniqueness of solutions for a class of nonlinear stochastic differential equations, which are not solved for the stochastic differential.

Statement of the Problem. Let us consider a nonlinear stochastic differential equation:

$$
d(x-G(t, x))=a(t, x) d t+\sigma(t, x) d W(t),
$$

which is not solved for the stochastic differential.
Here $x \in R^{d}, t \geq t_{0}, G(t, x)$, and $a(t, x)$ are $d$ dimensional vector functions; $\sigma(t, x)$ is a $d \times m$ matrix and $W(t)$ is an $m$-dimensional Wiener process with independent components. Let $x$ be a random vector in $R^{d}$, such that $E|x|^{2}<\infty$. Assume that $x$ does not depend on $W(t)-W\left(t_{0}\right)$, $F_{t}=\sigma\left\{x, W(s)-W\left(t_{0}\right), t_{0} \leq s \leq t\right\}$.

Definition 1. An $F_{t}$-dimensional process $x(t)$ is said to be the solution of (1) if $x\left(t_{0}\right)=x$ and

$$
\begin{aligned}
x-G(t, x(t))= & x-G\left(t_{0}, x\right) \\
& +\int_{t_{0}}^{t} a(s, x(s)) d s+\int_{t_{0}}^{t} \sigma(s, x(s)) d W(s)
\end{aligned}
$$

holds for every $t \geq t_{0}$ with probability 1 .

A solution $x(t)$ is said to be unique if for any continuous solutions $x(t), y(t)$ such that $x\left(t_{0}\right)=y\left(t_{0}\right)=x$ one has $P\left\{\sup _{s \in\left[t_{0}, t\right]}|x(s)-y(s)|>0\right\}=0$ for all $t \geq t_{0}$.

In this work we use the method of successive approximations to establish existence and uniqueness (pathwise) of the solution of (1). We study its probability properties. We prove that $x(t)$ is diffusion process and find coefficients of diffusion.

\section{Main Results}

Firstly we prove the theorem of existence and uniqueness of the solution. 
Theorem 2. Assume that $G(t, x)$ is a continuous function; $a(t, x), \sigma(t, x)$ are measurable functions for $x \in R^{d}, t \geq t_{0}$ and satisfy the following conditions:

(1) there exists a constant $C>0$, such that $|G(t, x)|+$ $|a(t, x)|+\|\sigma(t, x)\| \leq C(1+|x|)$ for $x \in R^{d}, t \geq t_{0} ;$

(2) there exist constants $L_{1}>0$ and $L>0$ such that $\left|G(t, x)-G\left(t, x^{\prime}\right)\right| \leq L_{1}\left|x-x^{\prime}\right|$ and

$$
\left|a(t, x)-a\left(t, x^{\prime}\right)\right|+\left\|\sigma(t, x)-\sigma\left(t, x^{\prime}\right)\right\| \leq L\left|x-x^{\prime}\right|
$$

$$
\text { for } x, x^{\prime} \in R^{d}, t \geq t_{0} .
$$

If $L_{1}<(1 / 6)^{3 / 4}$, then there exists a unique continuous solution $x(t)$ of (1) with probability 1 for all $t \geq t_{0}$. Moreover it has a bounded second moment such that $E|x(t)|^{2}<\infty$ for all $t \geq t_{0}$.

Proof. To find a solution of the integral equation (2), we use the method of successive approximations. We start by choosing an initial approximation $x_{0}(t)=x$.

At the next step,

$$
\begin{aligned}
x_{1}(t)= & x+G(t, x)-G\left(t_{0}, x\right) \\
& +\int_{t_{0}}^{t} a(s, x(s)) d s+\int_{t_{0}}^{t} \sigma(s, x(s)) d W(s) .
\end{aligned}
$$

Consequently,

$$
\begin{aligned}
x_{n+1}(t)=x & +G\left(t, x_{n}(t)\right)-G\left(t_{0}, x\right) \\
& +\int_{t_{0}}^{t} a\left(s, x_{n}(s)\right) d s+\int_{t_{0}}^{t} \sigma\left(s, x_{n}(s)\right) d W(s) .
\end{aligned}
$$

Consider an interval $\left[t_{0}, T\right]$ such that $B=3\left(L_{1}^{2}+5(T-\right.$ $\left.\left.t_{0}\right) L^{2}\right)<1$. Note that it can be always achieved by choosing a sufficiently small $T-t_{0}$ and by the conditions on $L_{1}$.

We prove that a solution exists on this interval.

From (4) it follows that

$$
\begin{gathered}
E\left|x_{1}(t)-x\right|^{2} \leq 3\left(E\left|G(t, x)-G\left(t_{0}, x\right)\right|^{2}+E\left|\int_{t_{0}}^{t} a(s, x) d s\right|^{2}\right. \\
\left.+\int_{t_{0}}^{t} E\|\sigma(s, x)\|^{2} d W(s)\right) \\
\leq 3\left(C_{1}\left(1+E|x|^{2}\right)+\left(T-t_{0}\right)^{2} C_{1}\left(1+E|x|^{2}\right)\right. \\
\left.+\left(T-t_{0}\right) C_{1}\left(1+E|x|^{2}\right)\right),
\end{gathered}
$$

where $C_{1}$ is a constant independent of $t_{0}, T$, and $x$.
Next, estimate

$$
E \sup _{t \in\left[t_{0}, T\right]}\left|x_{n+1}(t)-x_{n}(t)\right|^{2},
$$

$$
\begin{aligned}
& \left|x_{1}(t)-x\right|^{2} \\
& =\left|G(t, x)-G\left(t_{0}, x\right)+\int_{t_{0}}^{t} a(s, x) d s+\int_{t_{0}}^{t} \sigma(s, x) d W(s)\right|^{2} \\
& \leq 3\left[\left|G(t, x)-G\left(t_{0}, x\right)\right|^{2}+\left|\int_{t_{0}}^{t} a(s, x) d s\right|^{2}\right. \\
& \left.\quad+\left|\int_{t_{0}}^{t} \sigma(s, x) d W(s)\right|^{2}\right] \\
& \leq 3\left[C_{1}\left(1+|x|^{2}\right)\right. \\
& \left.+\left|\int_{t_{0}}^{t} a(s, x) d s\right|^{2}+\left|\int_{t_{0}}^{t} \sigma(s, x) d W(s)\right|^{2}\right] .
\end{aligned}
$$

Therefore,

$$
\begin{gathered}
E \sup _{t \in\left[t_{0}, T\right]}\left|x_{1}(t)-x\right|^{2} \\
\leq 3\left(C_{1}\left(1+E|x|^{2}\right)+E\left|\int_{t_{0}}^{T} a(s, x) d s\right|^{2}\right. \\
\left.+E \sup _{t \in\left[t_{0}, T\right]}\left\|\int_{t_{0}}^{t} \sigma(s, x(s)) d W(s)\right\|^{2}\right) \\
\leq 3\left(C_{1}\left(1+E|x|^{2}\right)+\left(T-t_{0}\right) \int_{t_{0}}^{T} C_{1}\left(1+E|x|^{2}\right) d s\right. \\
\left.\quad+4 \int_{t_{0}}^{T} C_{1}\left(1+E|x|^{2}\right) d s\right) \\
\leq 3\left(1+\left(T-t_{0}\right)^{2}+4\left(T-t_{0}\right)\right) C_{1}\left(1+E|x|^{2}\right) .
\end{gathered}
$$

The estimation of the stochastic integral follows from its properties. Similar to [11, p.20], we have

$$
\begin{aligned}
& E \sup _{t \in\left[t_{0}, T\right]}\left|x_{n+1}(t)-x_{n}(t)\right|^{2} \\
& \leq 3\left(L_{1}^{2} \sup _{t \in\left[t_{0}, T\right]}\left|x_{n}(t)-x_{n-1}(t)\right|^{2}\right. \\
& +\left(T-t_{0}\right) \int_{t_{0}}^{T} L^{2}\left|x_{n}(s)-x_{n-1}(s)\right|^{2} d s \\
& \left.+\sup _{t \in\left[t_{0}, T\right]}\left\|\int_{t_{0}}^{t}\left(\sigma\left(s, x_{n}(s)\right)-\sigma\left(s, x_{n-1}(s)\right)\right) d W(s)\right\|^{2}\right) \text {. }
\end{aligned}
$$


Thus, we have

$$
\begin{gathered}
E \sup _{t \in\left[t_{0}, T\right]}\left|x_{n+1}(t)-x_{n}(t)\right|^{2} \\
\leq 3\left(L_{1}^{2} E \sup _{t \in\left[t_{0}, T\right]}\left|x_{n}(t)-x_{n-1}(t)\right|^{2}\right. \\
+\left(T-t_{0}\right)^{2} L^{2} E \sup _{t \in\left[t_{0}, T\right]}\left|x_{n}(t)-x_{n-1}(t)\right|^{2} \\
\left.+4 \int_{t_{0}}^{T} L^{2} E\left|x_{n}(s)-x_{n-1}(s)\right|^{2} d s\right) \\
\leq 3\left(L_{1}^{2}+\left(T-t_{0}\right)^{2} L^{2}+4 L^{2}\left(T-t_{0}\right)\right) \\
\quad \times \sup _{t \in\left[t_{0}, T\right]}\left|x_{n}(s)-x_{n-1}(s)\right|^{2} \\
=B E \sup _{t \in\left[t_{0}, T\right]}\left|x_{n}(t)-x_{n-1}(t)\right|^{2} \\
\leq B^{n} E \sup _{t \in\left[t_{0}, T\right]}\left|x_{1}(t)-x\right|^{2} \\
\leq B^{n} 3\left(1+5\left(T-t_{0}\right)\right) C_{1}\left(1+E|x|^{2}\right) .
\end{gathered}
$$

Since $B<1$, it then follows that the series

$$
\begin{aligned}
\sum_{n=1}^{\infty} P & \left\{\sup _{t \in\left[t_{0}, T\right]}\left|x_{n+1}(t)-x_{n}(t)\right|>\frac{1}{n^{2}}\right\} \\
& \leq \sum_{n=1}^{\infty} B^{n} n^{4} 3\left(1+5\left(T-t_{0}\right)\right) C_{1}\left(1+E|x|^{2}\right)
\end{aligned}
$$

\section{converges.}

This implies the uniform convergence with probability 1 of the series

$$
x+\sum_{n=0}^{\infty}\left(x_{n+1}(t)-x_{n}(t)\right)
$$

on the interval $\left[t_{0}, T\right]$. Its sum is $x(t)$. So $x_{n}(t)$ converges to some random process. Every $x_{n}(t)$ is continuous with probability 1 . Whence it follows that the limit $x(t)$ is continuous with probability 1 , too.

Then prove uniqueness of this continuous solution. Assume that there exists a second continuous solution $y(t)$ of (1). Denote by $\varkappa_{N}(t)$ the random variable which equals 1 if $|x(s)| \leq N,|y(s)| \leq N$ and it equals 0 otherwise. Then

$$
\begin{array}{r}
\varkappa_{N}(t)(x(t)-y(t)) \\
=\varkappa_{N}(t)(G(t, x(t))-G(t, y(t))) \\
+\varkappa_{N}(t)\left[\int_{t_{0}}^{T} \varkappa_{N}(s)[a(s, x(s))-a(s, y(s))] d s\right. \\
+\int_{t_{0}}^{t} \varkappa_{N}(s)[\sigma(s, x(s)) \\
-\sigma(s, y(s))] d W(s)] .
\end{array}
$$

Therefore,

$$
\begin{aligned}
& E\left(\varkappa_{N}(t)|x(t)-y(t)|^{2}\right) \\
& \leq 3\left[E\left(\varkappa_{N}(t) L_{1}^{2}|x(t)-y(t)|^{2}\right)\right. \\
&+\left(t-t_{0}\right) \int_{t_{0}}^{t} E\left(\varkappa_{N}(s)|x(s)-y(s)|^{2}\right) d s \\
&\left.+\int_{t_{0}}^{t} E\left(\varkappa_{N}(s) L^{2}|x(s)-y(s)|^{2}\right) d s\right] .
\end{aligned}
$$

The last inequality implies the estimation

$$
\begin{aligned}
& \left(1-3 L_{1}^{2}\right) E\left(\varkappa_{N}(t)|x(t)-y(t)|^{2}\right) \\
& \quad \leq\left(1+\left(T-t_{0}\right)\right) L^{2} \int_{t_{0}}^{t} E\left(\varkappa_{N}(t)|x(s)-y(s)|^{2}\right) d s .
\end{aligned}
$$

By the Gronwall-Bellman inequality and the above inequality together, we have

$$
E\left(\varkappa_{N}(t)|x(t)-y(t)|^{2}\right)=0 .
$$

So,

$$
\begin{aligned}
P\{x(t) \neq y(t)\} \leq & P\left\{\sup _{s \in\left[t_{0}, t\right]}|x(s)|>N\right\} \\
& +P\left\{\sup _{s \in\left[t_{0}, t\right]}|y(s)|>N\right\} .
\end{aligned}
$$

Probabilities at the right-hand side of the above inequality approach to zero as $N \rightarrow \infty$, because $x(t)$ and $y(t)$ are continuous with probability 1 . Therefore $x(t)$ and $y(t)$ are stochastic equivalent.

We conclude that

$$
P\left\{\sup _{s \in\left[t_{0}, T\right]}|x(s)-y(s)|>0\right\}=0 .
$$

Hence, the existence and uniqueness of the solution are proved on $\left[t_{0}, T\right]$. 
Next, we show boundedness of a second moment of the solution. Denote again by $\varkappa_{N}(t)$ the indicator of the set

$$
\left\{\omega: \sup _{s \in\left[t_{0}, t\right]}|x(s)-x| \leq N\right\} .
$$

Then

$$
\begin{aligned}
\varkappa_{N}(t) & (x(t)-x) \\
= & \varkappa_{N}(t)\left(G(t, x(t))-G\left(t_{0}, x\right)\right) \\
& +\varkappa_{N}(t) \int_{t_{0}}^{t} \varkappa_{N}(s) a(s, x(s)) d s \\
& +\varkappa_{N}(t) \int_{t_{0}}^{t} \varkappa_{N}(s) \sigma(s, x(s)) d W(s) .
\end{aligned}
$$

Similarly, we have

$$
\begin{aligned}
& E\left(\varkappa_{N}(t)(x(t)-x)\right)^{2} \\
& \leq 3\left[E \varkappa _ { N } ( t ) \left(L_{1}|x(t)-x|\right.\right. \\
& \left.+|G(t, x)|+\left|G\left(t_{0}, x\right)\right|\right)^{2} \\
& +\left(T-t_{0}\right) \int_{t_{0}}^{t} E\left(\varkappa_{N}(s)(|a(s, x(s))-a(s, x)|\right. \\
& \left.+|a(s, x(s))|)^{2}\right) d s \\
& +\int_{t_{0}}^{t} E\left(\varkappa_{N}(s)(|\sigma(s, x(s))-\sigma(s, x)|\right. \\
& \left.\left.+|\sigma(s, x(s))|)^{2}\right) d s\right] \\
& \leq 9 L_{1}^{2} E\left(\varkappa_{N}(t)|x(t)-x|^{2}\right)+18 C_{1}\left(1+E|x|^{2}\right) \\
& +6\left(T-t_{0}\right) \int_{t_{0}}^{t} E\left(\varkappa_{N}(s) L^{2}|x(s)-x|^{2}\right) d s \\
& +6\left(T-t_{0}\right)^{2} C_{1}\left(1+E|x|^{2}\right) \\
& +6 \int_{t_{0}}^{t} E\left(\varkappa_{N}(s) L^{2}|x(s)-x|^{2}\right) d s \\
& +6\left(T-t_{0}\right)^{2} C_{1}\left(1+E|x|^{2}\right) .
\end{aligned}
$$

So,

$$
\begin{aligned}
(1- & \left.9 L_{1}^{2}\right) E\left(\varkappa_{N}(s)|x(s)-x|^{2}\right) \\
\leq & 18 C_{1}\left(1+E|x|^{2}\right)+12\left(T-t_{0}\right) C_{1}\left(1+E|x|^{2}\right) \\
& +\left(6 L^{2}\left(T-t_{0}\right)+6 L^{2}\right) \int_{t_{0}}^{t} E\left(\varkappa_{N}(s)|x(s)-x|^{2}\right) d s .
\end{aligned}
$$

Use the Gronwall-Bellman inequality to find the estimation

$$
E \varkappa_{N}(t)|x(t)-x|^{2} \leq D\left(1+E|x|^{2}\right),
$$

where $D$ is a constant independent of $N$. Applying the Fatou lemma to the last inequality and assuming that $N \rightarrow \infty$, we have

$$
E|x(t)-x|^{2} \leq D\left(1+E|x|^{2}\right) .
$$

Since $E|x|^{2} \leq \infty$, then it follows from [8] that $E|x|^{2}<\infty$ for every $t \in\left[t_{0}, T\right]$.

Thus existence, uniqueness, and boundedness of the second moment of the solution $x(t)$ are proved on $\left[t_{0}, T\right]$. Since the constant $B$ is dependent only on $T-t_{0}$ and $E|x|^{2}<$ $\infty$, and $x(T)$ is independent of $W(s)-W(T)$ for $s \geqslant T$, then by similar manner we can prove the existence and uniqueness of the solution of the IVP with initial conditions $(T, x(T))$ on the interval $\left[T, T_{1}\right]$, where $T_{1}$ is chosen such that the inequality $3\left(L_{1}^{2}+5\left(T_{1}-T\right) L^{2}\right)<1$.

This procedure can be repeated in order to extend the solution of (1) to the entire semiaxis $t \geqslant t_{0}$. The theorem is proved.

Notes. The existence and uniqueness of the solution can be obtained as corollary from work [1], where this result was proved for an SDE of neutral type by replacing a condition for Lipschitz constant $L_{1}<(1 / 6)^{3 / 4}$ with more weak condition $L_{1}<1$. But by using our method, paths of obtained solution are continuous with probability 1 . Otherwise, in the pointed work only the measurability of the solution and boundedness of its second moment were stated.

Now, we state some probability properties of the solution obtained in Theorem 2 . We prove that under assumptions of Theorem 2, the solution of (1) is a Markov random process. Moreover, if the coefficients are continuous then it is a diffusion process. We will find its diffusion coefficients.

Theorem 3. Under conditions (1)-(2) of Theorem 2 the solution $x(t)$ of $(1)$ is a Markov process with a transition probability defined by

$$
P(t, x, s, A)=P\left\{x_{t, x}(s) \in A\right\},
$$

where $x_{t, x}(s)$ is a solution of that equation

$$
\begin{aligned}
x_{t, x}(s)= & G\left(s, x_{t, x}(s)\right)+x-G(t, x) \\
& +\int_{t}^{s} a\left(u, x_{t, x}(u)\right) d u \\
& +\int_{t}^{s} \sigma\left(u, x_{t, x}(u)\right) d W(u),
\end{aligned}
$$

where $s \geqslant t \geqslant t_{0}, x \in R^{d}$.

Proof. As in Theorem 2, we solve (1) by the method of successive approximations. It can be shown that $x_{t, x}(s)$ is completely defined by the nonrandom initial value $x$ and the 
process $W(s)-W(t)$ for $s>t$, which are independent of $F_{t}$. Since $x(t)$ is a solution of (2), it is $F_{t}$ measurable. Hence $x_{t, x}(s)$ is independent of $x(t)$ and events from $F_{t}$. Note that, from the uniqueness of the solution $x(s)$ for $s>t$, it follows that it is a unique solution of the equation

$$
\begin{aligned}
x(s)= & G(s, x(s)) \\
& +x(t)-G(t, x(t))+\int_{t}^{s} a(u, x(u)) d u \\
& +\int_{t}^{s} \sigma(u, x(u)) d W(u) .
\end{aligned}
$$

Since the process $x_{t, x(t)}(s)$ is also a solution of this equation, then $x(s)=x_{t, x(t)}(s)$ with probability 1 .

As for the rest, the proof is the same as the proof of the theorem for ordinary stochastic equations [11]. The theorem is proved.

We have a corollary from this theorem.

Corollary 4. Suppose that conditions of Theorem 2 are satisfied. Then

(1) if functions $G, a$, and $\sigma$ are independent of $t$, then the solution $x(t)$ is a homogeneous Markov process;

(2) if functions $G, a$, and $\sigma$ are periodic functions with period $\theta$, then a transition probability is periodic function; that is, $P(t+\theta, x, s+\theta, A)=P(t, x, s, A)$.

Now, we investigate conditions for which the solution of (1) is a diffusion process. For this we must find an additional estimate.

Lemma 5. Let $x_{t, x}(s)$ be a solution of (2) such that $x_{t, x}(t)=$ $x, x \in R^{d}$. If conditions of Theorem 2 are satisfied and $G_{t}(t, x)$ is continuous for $t \geq t_{0}, x \in R^{d}$, then an inequality

$$
E\left|x_{t, x}(s)-x\right|^{4} \leq H(s-t)^{2}\left(1+|x|^{4}\right)
$$

holds. Here $H>0$ is a constant dependent only on $C, L_{1}, L$, and $G_{t}(t, x)$.

Proof. Denote by $\varkappa_{N}(s)$ the indicator of the set

$$
\left\{\omega: \sup _{u \in[t, s]}\left|x_{t, x}(u)-x\right| \leq N\right\} .
$$

Similarly to finding estimation (24), we have

$$
\begin{aligned}
& E\left(\varkappa_{N}(s)\left|x_{t, x}(s)-x\right|^{4}\right) \\
& \quad \leq 27 E\left(\varkappa _ { N } ( s ) \left(G\left(s, x_{t, x}(s)\right)-G(s, x)\right.\right. \\
& \left.+G(s, x)-G(t, x))^{4}\right)
\end{aligned}
$$

$$
\begin{aligned}
& +27 E\left|\int_{t}^{s} \varkappa_{N}(u) a\left(u, x_{t, s}(u)\right) d u\right|^{4} \\
& +27 E\left|\int_{t}^{s} \varkappa_{N}(u) \sigma\left(u, x_{t, x}(u)\right) d W(u)\right|^{4} \\
& \leq 6^{3} L_{1}^{4} E\left(\varkappa_{N}(s)\left|x_{t, x}(s)-x\right|^{4}\right) \\
& +6^{3}\left|G_{t}^{4}(t, x)\right|(s-t)^{4}+o_{x}(t-s)^{4} \\
& +27(s-t)^{3} \int_{t}^{s} E \varkappa_{N}(u)\left|a\left(u, x_{t, x}(u)\right)\right|^{4} d u \\
& +(27)(36)(s-t) \int_{t}^{s} E \varkappa_{N}(u)\left|\sigma\left(u, x_{t, x}(u)\right)\right|^{4} d u,
\end{aligned}
$$

where $o_{x}$ points dependence on $x$.

The last estimate follows from Hölder's inequality and properties of stochastic integral. From inequality (30) and condition for $L_{1}$, we have

$$
\begin{aligned}
(1- & \left.6^{3} L_{1}^{4}\right) E\left(\varkappa_{N}(s)\left|x_{t, x}(s)-x\right|^{4}\right) \\
\leq & 6^{3}\left|G_{t}(t, x)\right|^{4}(s-t)^{4}+o_{x}(t-s)^{4} \\
& +(27)(8)(s-t)^{3} \int_{t}^{s} L^{4} E\left(\varkappa_{N}(u)\left|x_{t, x}(u)-x\right|^{4}\right) d u \\
& +(27)(8)(s-t)^{3} \int_{t}^{s}|a(u, x)|^{4} d u \\
& +(27)(36)(8)(s-t) \int_{t}^{s}|\sigma(u, x)|^{4} d u \\
& +(27)(36)(8)(s-t) L^{4} \int_{t}^{s} E\left(\varkappa_{N}(u)\left|x_{t, x}(u)-x\right|^{4}\right) d u \\
\leq & 6^{3}\left|G_{t}(t, x)\right|^{4}(s-t)^{4}+o_{x}(s-t)^{4} \\
& +6^{3}(s-t)^{4} C_{2}\left(1+|x|^{4}\right)+6^{5}(s-t)^{2} C_{2}\left(1+|x|^{4}\right) \\
& +6^{5}(s-t) L^{4} \int_{t}^{s} E\left(\varkappa_{N}(u)\left|x_{t, s}(u)-x\right|^{4}\right) d u,
\end{aligned}
$$

where $C_{2}$ depends only on $C$.

Further, the right-hand side of (31) can be estimated by

$$
\left(1+|x|^{4}\right) R(s-t)^{2}+R_{1} \int_{t}^{s} E\left(\varkappa_{N}(u)\left|x_{t, x}(u)-x\right|^{4}\right) d u,
$$

where $R=6^{3}\left|G_{t}(t, x)\right|^{4}+6^{3} C_{2}+6^{5} C_{2}+1$ and $R_{1}=6^{5} L^{4}, t \leq$ $s \leq t+1$.

Using the lemma from [11, p.38], we obtain

$$
E \varkappa_{N}(u)\left|x_{t, x}(u)-x\right|^{4} \leq H(s-t)^{2}\left(1+|x|^{4}\right),
$$

where $H>0$ depends only on $C_{2}, L$, and $G_{t}(t, x)$.

Now, assume that $N \rightarrow \infty$ and obtain estimate (28). This completes the proof of the Lemma. 
Theorem 6. If conditions (1)-(2) of Theorem 2 are satisfied and functions $a(t, x), \sigma(t, x), G(t, x), G_{t}(t, x), G_{x_{i}}(t, x)$, and $G_{x_{i} x_{j}}(t, x), i, j=1, \bar{d}$, are continuous for $t \geqslant t_{0}, x \in R^{d}$. Functions $G_{t}(t, x), G_{x_{i}}(t, x)$, and $G_{x_{i} x_{j}}(t, x)$ satisfy Lipschitz condition with respect to $x$ in neighborhood of every point $(t, x)$. Then the solution of (1) is diffusion process with

$$
\begin{gathered}
\bar{a}(t, x)=\left(I-G_{x}(t, x)\right)^{-1} \\
\times\left[G_{t}(t, x)+a(t, x)+\frac{1}{2} G_{x x}(t, x)\left(I-G_{x}(t, x)\right)^{-1}\right. \\
\left.\quad \times \sigma(t, x) \sigma^{T}(t, x)\left(\left(I-G_{x}(t, x)\right)^{T}\right)^{-1}\right]
\end{gathered}
$$

and diffusion matrix with

$$
\bar{\sigma}(t, x)=\left(I-G_{x}(t, x)\right)^{-1} \sigma(t, x) \sigma^{T}(t, x)\left(\left(I-G_{x}(t, x)\right)^{T}\right)^{-1} .
$$

Proof. Take any point $(t, x)$ from a region $t \geqslant t_{0}, x \in R^{d}$. Consider a stochastic ITO equation in a closed neighborhood of this point

$$
\begin{aligned}
d y=( & \left.I-G_{x}(s, y)\right)^{-1} \\
\times & \quad\left[G_{t}(s, y)+a(s, y)+\frac{1}{2} G_{x x}(s, y)\left(I-G_{x}(s, y)\right)^{-1}\right. \\
& \left.\quad \times \sigma(s, y) \sigma^{T}(s, y)\left(\left(I-G_{x}(s, y)\right)^{T}\right)^{-1}\right] d s \\
& +\left(I-G_{x}(s, y)\right)^{-1} \sigma(s, y) d W(s) .
\end{aligned}
$$

From the conditions of the theorem, it follows that there exists a closed neighborhood of the point $(t, x)$, such that coefficients of the equation are Lipschitz with respect to $x$. Fix this neighborhood. Extend these coefficients on the all region $t \geq t_{0}, x \in R^{d}$ such that they remain continuous by both variables, Lipschitz and linear with respect to $x$.

Then the equation

$$
d y=a_{1}(s, y) d s+\sigma_{1}(s, y) d W(s)
$$

with these extended coefficients has a unique solution of the IVP $y(t)=x$ for $s>t$.

From ITO formula and coincidence of coefficients of (36) and (37) in this neighborhood of the point $(t, x)$, one can show that processes $x_{t, x}$ and $y(s)$ coincide in this neighborhood.

It is known that under these assumptions the process $y(s)$ is diffusion. Its diffusion coefficients in the point $(t, x)$ are defined by coefficients of (36).
Next, show that $x(s)$ has the same diffusion coefficients. To do this it is sufficient to estimate the following limits and use estimation (28)

$$
\begin{gathered}
\lim _{s \rightarrow t} \frac{1}{s-t} \int_{|y-x| \leq \varepsilon_{0}}(y-x) P(t, x, s, d y), \\
\lim _{s \rightarrow t} \frac{1}{s-t} \int_{|y-x| \leq \varepsilon_{0}}(z, y-x)^{2} P(t, x, s, d y),
\end{gathered}
$$

where $\varepsilon_{0}$ is chosen such that the point $(s, y)$ is laying in this neighborhood.

We get

$$
\begin{aligned}
& \lim _{s \rightarrow t} \frac{1}{s-t} \int_{|y-x| \leq \varepsilon_{0}}(y-x) P(t, x, s, d y) \\
& =\lim _{s \rightarrow t} \frac{1}{s-t} \int_{\left\{w:\left|x_{t, x}(s)-x\right| \leq \varepsilon_{0}\right\}}\left(x_{t, x}(s)-x\right) P(d w) \\
& =\lim _{s \rightarrow t} \frac{1}{s-t} \int_{\left\{w:|y(s)-x| \leq \varepsilon_{0}\right\}}(y(s)-x) P(d w) \\
& =\left(I-G_{x}(t, x)\right)^{-1}\left[G_{t}(t, x)+a(t, x)+\frac{1}{2} G_{x x}(t, x)\right. \\
& \quad \times\left(I-G_{x}(t, x)\right)^{-1} \sigma(t, x) \\
& \left.\quad \times \sigma^{T}(t, x)\left(\left(I-G_{x}(t, x)\right)^{T}\right)^{-1}\right] .
\end{aligned}
$$

Similarly, obtain the second limit in (38):

$$
\begin{gathered}
\lim _{s \rightarrow t} \frac{1}{s-t} \int_{|y-x| \leq \varepsilon_{0}}(z, y-x)^{2} P(t, x, s, d y) \\
=\left(\left(I-G_{x}(t, x)\right)^{-1} \sigma(t, x) \sigma^{T}(t, x)\right. \\
\left.\quad \times\left(\left(I-G_{x}(t, x)\right)^{T}\right)^{-1} z, z\right) .
\end{gathered}
$$

So, the existence of limits in (38) is proved for every point $(t, x)$ and fixed $\varepsilon_{0}$, chosen for this point. It is clear that from (28), these limits exist for every $\varepsilon>0$ and they are independent of $\varepsilon$. From the above and the definition of diffusion process $[12$, p.67] we can finish the proof. The theorem is proved.

\section{References}

[1] V. B. Kolmanovskii and V. R. Nosov, Stability and Periodic Modes of Control System with Aftereffect, Nauka, Moscow, Russia, 1981.

[2] V. Kolmanovskii, N. Koroleva, T. Maizenberg, X. Mao, and A. Matasov, "Neutral stochastic differential delay equations with Markovian switching," Stochastic Analysis and Applications, vol. 21, no. 4, pp. 819-847, 2003.

[3] X. Mao, Stochastic Differential Equations and Their Applications, Horwood, Chichester, UK, 1997.

[4] X. X. Liao and X. Mao, "Exponential stability in mean square of neutral stochastic differential difference equations," Dynamics of Continuous, Discrete and Impulsive Systems, vol. 6, no. 4, pp. 569-586, 1999. 
[5] X. Mao, "Asymptotic properties of neutral stochastic differential delay equations," Stochastics and Stochastics Reports, vol. 68, no. 3-4, pp. 273-295, 2000.

[6] A. M. Samoilenko, N. I. Mahmudov, and O. M. Stanzhytskii, "Existence, uniqueness, and controllability results for neutral FSDES in Hilbert spaces," Dynamic Systems and Applications, vol. 17, no. 1, pp. 53-70, 2008.

[7] T. E. Govindan, "Stability of mild solutions of stochastic evolution equations with variable delay," Stochastic Analysis and Applications, vol. 21, no. 5, pp. 1059-1077, 2003.

[8] Y. Boukfaoui and M. Erraoui, "Remarks on the existence and approximation for semilinear stochastic differential equations in Hilbert spaces," Stochastic Analysis and Applications, vol. 20, no. 3, pp. 495-518, 2002.

[9] N. I. Mahmudov, "Existence and uniqueness results for neutral SDEs in Hilbert spaces," Stochastic Analysis and Applications, vol. 24, no. 1, pp. 79-95, 2006.

[10] V. B. Kolmanovskiĭ and L. E. Shaŭkhet, Control of Systems with Aftereffect, vol. 157, American Mathematical Society, Providence, RI, USA, 1996.

[11] I. I. Gikhman and A. V. Skorokhod, Stochastic Differential Equations, Naukova Dumka, Kyiv, Ukrania, 1968.

[12] I. I. Gikhman and A. V. Skorokhod, Introduction in Theory of Random Processes, Naukova Dumka, Kyiv, Ukrania, 1977. 


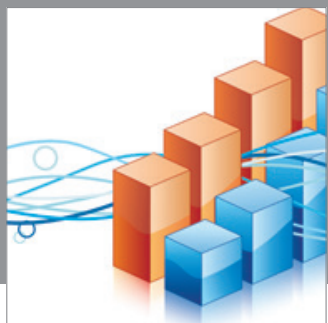

Advances in

Operations Research

mansans

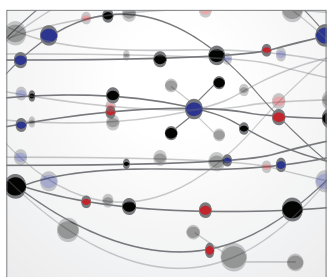

The Scientific World Journal
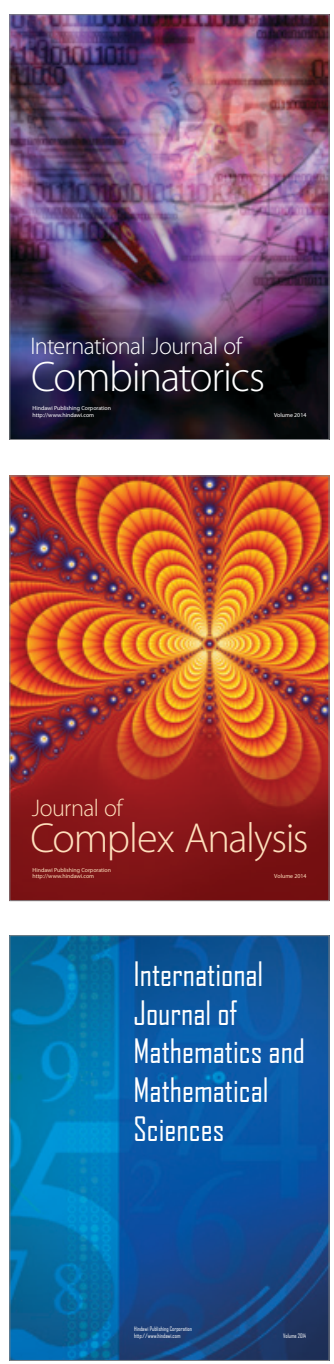
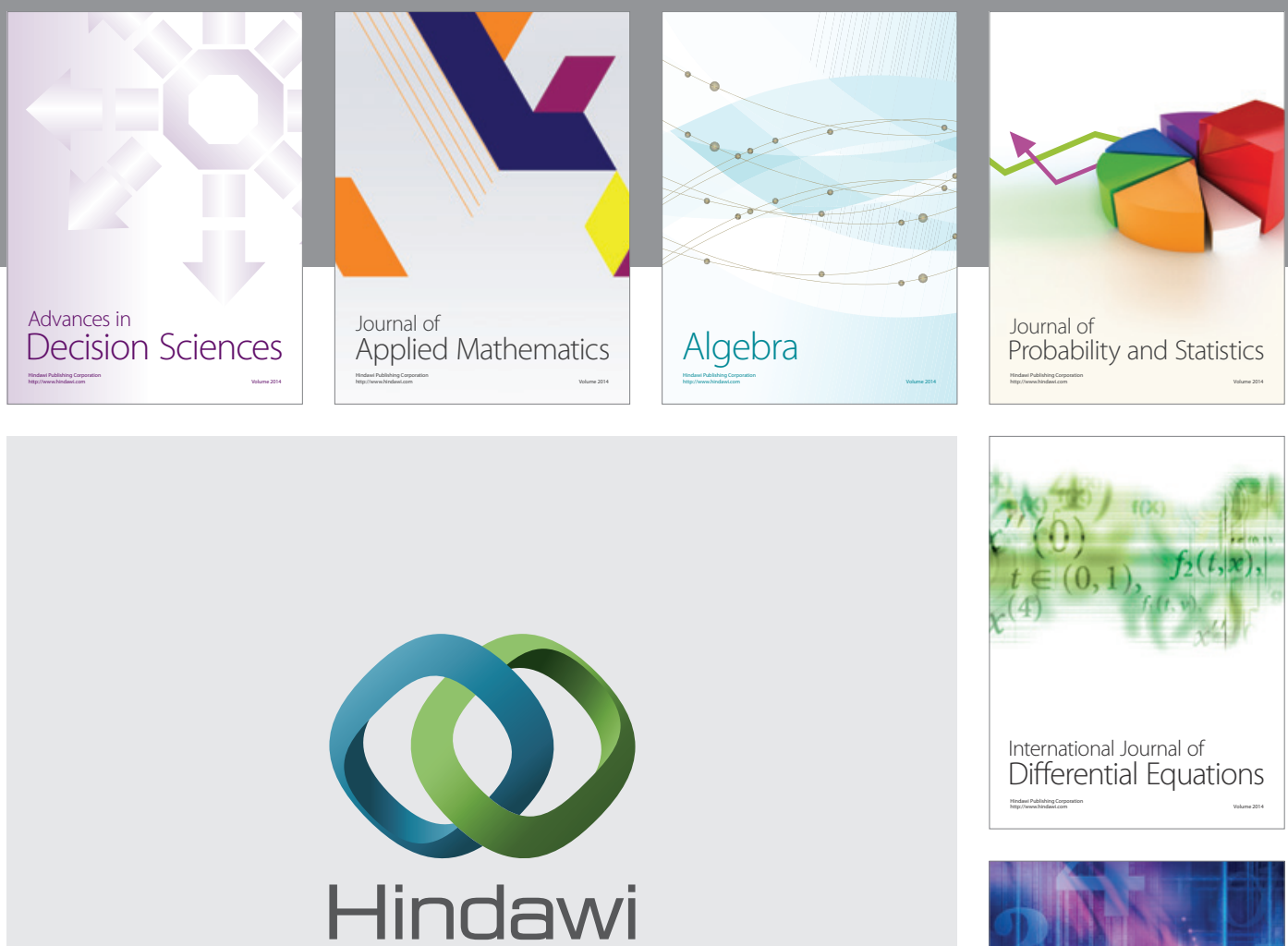

Submit your manuscripts at http://www.hindawi.com
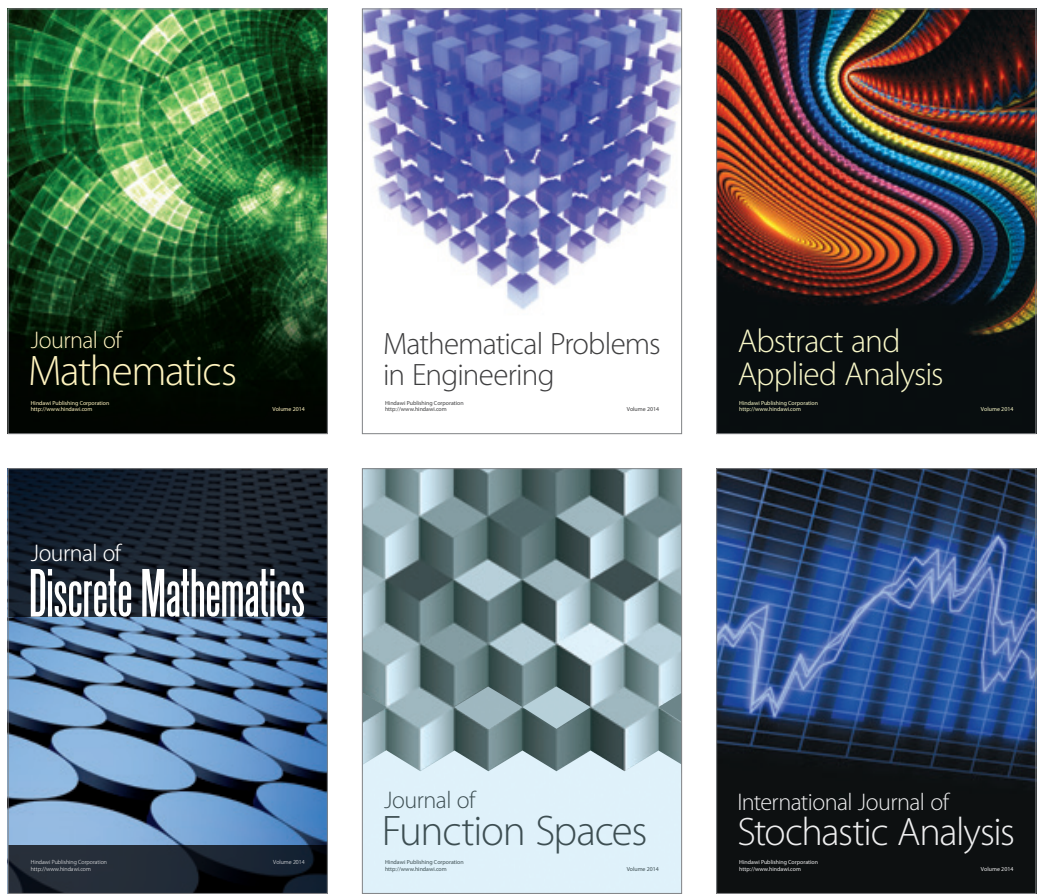

Journal of

Function Spaces

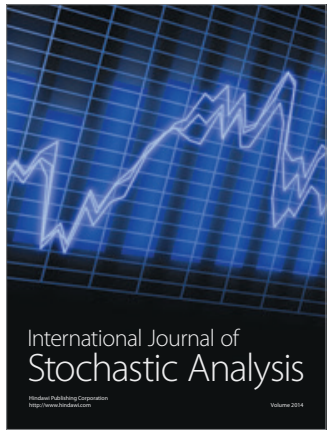

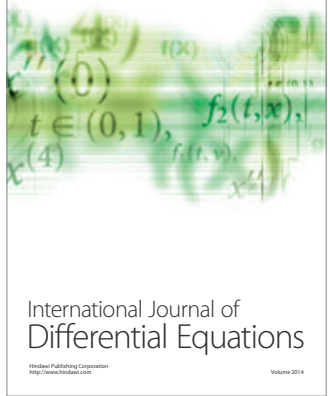
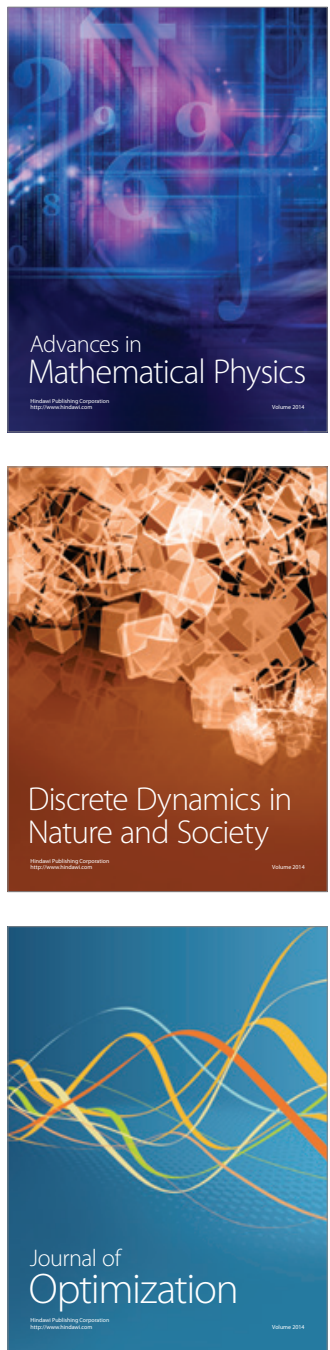